\title{
MODELO CONCEPTUAL DE UN SISTEMA DE INFORMACION DOCUMENTAL
}

\section{Codina *}

Resumen: Según el modelo conceptual que se expone aquí, un sistema de información documental es un sistema de información especializado en la explotación del conocimiento, concretamente del conocimiento registrado en documentos cognitivos. Tal clase de sistemas aceptan documentos y necesidades de información como entradas $y$, tras un proceso de transformación, producen personas informadas como salida.

Palabras clave: Sistemas de información documentales, modelos conceptuales, documentos cognitivos, recuperación de información.

\begin{abstract}
According to the conceptual model explained in this paper, a document-based information system is an information system devoted to exploit the knowledge recorded in cognitive documents. This kind of system accepts cognitive documents and information needs as inputs, and can produce informed people as output.

Keywords: Document-based information systems, Conceptual models, Cognitive documents, Information retrieval.
\end{abstract}

\section{Introducción}

Un modelo conceptual expresa los elementos fundamentales de un sistema, haciendo abstracción de los detalles particulares de las diversas ocurrencias de los sistemas reales existentes. El objetivo de un modelo conceptual es proporcionar una representación del objeto o fenómeno que permita su comprensión global, como un todo, sin que tal comprensión quede dificultada por la confusión entre las cuestiones accidentales, que sólo se dan en algunas ocurrencias del modelo.

Los modelos conceptuales también facilitan la comunicación entre equipos de personas implicadas en tareas comunes, al eliminar la ambiguiedad de ciertos aspectos del fenómeno considerado, y posibilitar el uso de un lenguaje común entre los miembros del equipo que evita los malentendidos, especialmente dados a aparecer, paradójicamente, en todos los. asuntos relacionados con el desarrollo de sistemas de información.

Los aspectos básicos del modelo que aquí se presenta se han tomado, principalmente, de la teoría de sistemas, particularmente de Bertalanffy (1986), Bunge (1993), y Checkland (1981); del método de Yourdon (1993) de análisis estructurado, de la obra de Walker (1991) sobre sistemas de información mediante ordenadores, de la propuesta de Soergel (1985) de un modelo general de un sistema de recuperación de información, que ha sido seguida y ligeramente reelaborada por Willitts (1992), así como de algunos autores que relacionan la teoría de sistemas con los sistemas de información documentales (Baiget, 1986; Currás, 1988).

* Universidad Pompeu Fabra. Barcelona.

Recibido: 15-5-94. 
Además, de Yourdon se adopta la diferencia entre sistemas «que son interesantes porque hacen cosas» y sistemas "que son depósitos interesantes de información», por decirlo de la forma más parecida posible a su propio estilo. De Checkland se toma su particular concepción de la teoría de sistemas aplicada a la resolución de problemas y su concepto de sistemas de actividades humanas, ambos recogidos en su Soft System Methodology.

\section{Los sistemas de información en general}

Siguiendo a Walker (1991), un sistema de información es un sistema que mantiene alguna clase de registros sobre las características y la evolución de otro sistema, el cual se suele designar como sistema del mundo real, o como sistema objeto (Walker, 1991).

El sistema de información no registra todas las variables y parámetros posibles del sistema del mundo real (o sistema objeto) que son virtualmente infinitos, sino que abstrae y selecciona ciertas características del sistema objeto, de acuerdo con los propósitos del sistema de información y con los intereses de sus usuarios.

Por tanto, un sistema de información de alguna manera es un modelo de una parte del mundo real (Yourdon, 1993). Ahora bien, aunque el modelo construido por el sistema de información debe obedecer a los propósitos particulares del sistema y a los intereses de sus usuarios, también debe ser fiel al sistema objeto para ser eficiente.

Siguiendo a Yourdon (1993), existe una clase particular de sistemas objeto que pueden resultar de interés, no tanto por las acciones que llevan a cabo, sino por tratarse de depósitos de conocimiento.

Por tanto, podemos considerar que existen dos grandes clases de sistemas del mundo real que pueden ser modelados por los sistemas de información:

1. Sistemas de actividades humanas, o sistemas "que hacen cosas».

2. Sistemas o «depósitos» de conocimiento.

Un ejemplo típico de un sistema de actividades humanas es una unidad de producción, pero también una universidad, un departamento de la Administración o un hospital, etc.

Un ejemplo típico de un sistema de conocimiento es una biblioteca o un centro de documentación, pero también un conjunto de documentos electrónicos distribuidos en una red de ordenadores.

En un sistema de actividades humanas, el aspecto esencial son las actividades del sistema, y los datos que memoriza el sistema son datos necesarios para que el sistema desarrolle correctamente sus actividades, incluyendo los mecanismos de control, etc.

Sin embargo, tales datos no tienen importancia intrínseca porque, aunque el sistema de información en sí tenga un valor estratégico, los datos concretos sólo tendrán un valor táctico en función de las actividades diarias del sistema.

En cambio, en un sistema de conocimiento la situación es simétricamente inversa. Las actividades del sistema tienen poca importancia, porque no son especialmente sofisticadas, pero el tipo de datos es esencial, porque se trata, de hecho, de conocimiento registrado, es decir, algo más que meros datos. 
El punto importante aquí es el siguiente: la existencia de estas dos clases de sistemas deberá ser tenida en cuenta a la hora de elegir las herramientas conceptuales de análisis y diseño del sistema; así como, posteriormente, las herramientas físicas de implantación.

De aquí la perplejidad de quienes intentan aplicar herramientas de modelado sin tener en cuenta tal diferencia. Por ejemplo, mientras el modelo entidad-relación $^{1}$ es especialmente útil para modelar sistemas de actividades (Jackson, 1990), no suele aportar nada a los sistemas de recuperación de información documentales, porque, por ejemplo, ¿qué relación existe entre un artículo y una monografía, o qué relación existe entre un documento y sus descriptores, desde el punto de vista del citado modelo? y, sobre todo, ¿qué aporta al diseño de un sistema de recuperación de información documental?

A la inversa, el modelo de representación de la información basado en descriptores y en lenguajes documentales es imprescindible en sistemas de recuperación de información, pero no aporta nada, salvo ruido y confusión, a un sistema de gestión de documentos administrativos. Por ejemplo: ¿qué sentido tiene describir y asignar descriptores a un documento administrativo ${ }^{2}$ o a un asiento contable?

Sin embargo, sería útil y necesaria la existencia de sistemas integrados de información capaces de soportar ambas clases de sistemas, particularmente en un momento en que ello empieza a ser también tecnológicamente viable (Canals, 1994); pero, para integrar bien es cuando se hace más necesario que nunca comprender la naturaleza diferenciada de aquello que se desea integrar en un todo armónico.

\section{Modelo conceptual de un sistema de información documental}

A partir de los conceptos generales de sistemas de información examinados anteriormente, los elementos que forman parte del modelo conceptual de un sistema de información documental que se propone aquí son los siguientes:

1. Un conjunto de conceptos fundamentales y, en su caso, unas proposiciones asociadas.

2. Un modelo general de un sistema de información documental.

3. Un modelo general de los procesos de transformación que tienen lugar en el interior del sistema.

4. Una proposición general sobre la Documentación y dos proposiciones particulares, relativas al estatuto de la Documentación como disciplina científica y a su relación con otras disciplinas cuyo objeto de estudio también es la información en alguna de sus facetas.

1 Como es sabido, el modelo entidad-relación, desarrollado por Chen, permite representar las distintas entidades que intervienen en una actividad y las relaciones que mantienen entre ellas, de cara a transformar las entidades y las relaciones en sendas tablas capaces de soportar las operaciones del álgebra relacional.

${ }^{2}$ Como es sabido, la gestión de documentos administrativos obedece a una lógica completamente distinta. Se rige, entre otros, por los principios de gestión a nivel de series documentales, cuadros de clasificación, ciclo de vida de los documentos y por el así llamado "respeto por el origen». Ver, por ejemplo, Heredia, A. Archivistica general: teoria y prúctica. Sevilla: Diputación de Sevilla, 1991. 


\section{Conceptos fundamentales}

Los conceptos fundamentales que ayudan a construir el modelo son los siguientes:

01. Sistema.

02. Sistema de información.

03. Sistema objeto.

04. Sistema determinista y sistema probabilista.

05. Entidad.

06. Documento.

07. Fondo documental no trivial.

08: Documento cognitivo.

09. Relevancia de un documento.

10. Necesidad de información.

11. Entorno de un sistema.

Sistema: conjunto de elementos en interacción con un propósito u objetivo común. Todo sistema puede caracterizarse por su entorno, su propósito, sus entradas, sus salidas y los procesos que transforman las entradas en salidas.

Sistema de información: Un sistema que mantiene registros sobre las características y la evolución de otro sistema, denominado sistema objeto. Un sistema de información posee los elementos necesarios para capturar, almacenar, represen$\operatorname{tar}$ y recuperar información. Todo sistema de información acepta una clase de datos como entrada, los somete a algunas transformaciones y proporciona información a unos usuarios.

Proposición: Puesto que manipula símbolos (señales de cualquier naturaleza, pero frecuentemente cadenas de caracteres y números) un sistema de información es un sistema de procesamiento de símbolos o, más abreviadamente, un sistema simbólico.

Sistema objeto: un sistema físico o conceptual sobre el cual un sistema simbólico mantiene registros para representar sus diversos estados y atributos.

Proposición asociada: Los sistemas objeto deben estar formados por entidades reconocibles e identificables para que otro sistema pueda mantener información sobre ellos (Yourdon, 1993).

Sistema determinista y sistema probabilista: En un sistema determinista es posible predecir una salida dada una entrada. En un sistema probabilista es imposible predecir una salida dada una entrada, y todo lo que puede hacerse es estimar el grado de probabilidad de que se produzca una salida determinada.

Entidad: Un elemento de un sistema objeto. Puesto que son identificables, estas entidades deben poseer algunos atributos que las caracterizan inequivocamente. Por ejemplo, para el SI de un museo, los visitantes no pueden ser entidades, ya que el sistema no posee ningún medio para identificarlos (al margen de que, probablemente, no estará interesado en hacerlo). Las entidades podrían ser, en cambio, las obras expuestas o en depósito, los documentos de su centro de documentación, etc.

Documento: Un conjunto de señales coherentes, registradas en un soporte material, realizadas con intención de comunicar y que, en su forma final, pueden ser interpretadas por un ser humano. 
Proposición: En tanto las señales siguen una gramática socialmente aceptada y dan a conocer algún aspecto de la realidad física o conceptual, los documentos son, desde un punto de vista antropológico, conocimiento o información registrada.

Fondo documental no trivial: Un fondo documental es no trivial cuando está formado por un gran número de documentos y cuando está formado por documentos cognitivos.

Documento cognitivo: Documento que recoge un trabajo científico, técnico o cultural y que, a diferencia de otros documentos, por ejemplo, los documentos administrativos de una empresa particular, es susceptible de interesar a la humanidad en su conjunto (Van Slype, 1988).

Relevancia de un documento: La capacidad de un documento para satisfacer una necesidad de información dada. Un documento puede tener un grado de relevancia igual a 0 (totalmente irrelevante), igual a 1 (totalmente relevante), o cualquier valor intermedio. Puede hablarse también de documento relevante.

Proposición: La relevancia de un documento es una coproducción entre el documento y la necesidad de información, por tanto, es una cualidad del documento, pero es dinámica y es diferente para cada usuario.

Necesidad de información: Estado subjetivo que experimenta un sujeto cuando detecta un estado anómalo en sus conocimientos (Belkin, citado por Ellis, 1990). Las necesidades de información no son directamente observables.

Proposición: La eficacia de un SI documental depende de la habilidad del SI documental para sugerir a los usuarios representaciones adecuadas de sus necesidades de información.

Entorno de un sistema: Todos los sistemas existen en un medio determinado que los contiene y con el que mantiene relaciones. Ahora bien, las relaciones que mantiene el sistema con su entorno son más débiles que las que mantienen los elementos del sistema entre sí. Se considera que un elemento forma parte del entorno y no del sistema, si el elemento (que puede ser otro sistema), pese a mantener relaciones con el sistema, escapa a su control (Van Gigch, 1987).

Proposición: En un sistema de información documental, los usuarios son la parte más importante del entorno. Se considera que forman parte del entorno y no del sistema porque los usuarios no pueden ser controlados por el sistema, por lo menos en el mismo sentido en que son controlados los documentos.

\section{Modelo general de un sistema de información documental}

Con los elementos precedentes puede construirse la siguiente definición:

Sistema de información documental: Un sistema de información documental es un sistema de información que acepta documentos y necesidades de información como entradas y produce personas informadas como salida (Soergel, 1985; Willitts, 1992).

El propósito de un sistema de información documental es explotar la información y el conocimiento registrado en los documentos para ponerlos al servicio de los usuarios (Morón, 1988). 


\section{Procesos de transformación del sistema}

El proceso de transformación que tiene lugar en el sistema se realiza en el subsistema de recuperación de información, mediante dos funciones principales (que, a su vez, se componen de diversas subfunciones que no se estudiarán aquí con detalle):

1. Función de representación.

2. Función de comparación.

La función de representación deriva representaciones formales (RFs) de los documentos y de las necesidades de información.

La función de comparación compara RFs de documentos y RFs de necesidades de información y selecciona los documentos que presentan un mayor grado de similitud con la necesidad de información.

La hipótesis subyacente es que los documentos relevantes poseen una RF parecida a la $\mathrm{RF}$ de la necesidad de información. Cuanto más relevante sea un documento, más se parecerán sus RFs.

\section{Representación formal de documentos y de necesidades de información}

La función de representación se resuelve mediante una serie de operaciones que se conocen con el nombre de cadena documental, y que no detallaremos aquí por existir abundante documentación sobre ella (ver, por ejemplo: Guinchat y Menou, 1992).

Un producto resultante de la cadena documental son las representaciones formales de documentos, también llamadas documentos secundarios, formadas por una descripción documental y por un conjunto de términos de indización.

Otro producto resultante son las representaciones de las necesidades de información, también llamadas preguntas, en forma de una ecuación de búsqueda, formada por términos de indización y, eventualmente, combinados mediante operadores booleanos o de proximidad. En ambas representaciones, en situación ideal, los términos de indización se extraen de un lenguaje común de indización (tesauros).

\section{La función de comparación}

En un SID, por tanto, existen dos objetos, la entidad documento que se representa mediante un conjunto de términos de indización $\left(t_{1}, t_{2}, \ldots t_{n}\right)$ que expresan los diversos temas contenidos en el documento $(\mathrm{A}, \mathrm{B}, \mathrm{C} . .$.$) , y el objeto pregunta, que$ se representa también por otro conjunto de términos de indización $\left(t_{1}, t_{2}, \ldots t_{n}\right)$ que representan a los diversos temas que expresan la necesidad de información.

Dados $n$ únicos términos de indización, tanto los documentos como las preguntas pueden concebirse como vectores formados por uno de los dos valores posibles que puede adquirir cada uno de los términos: 1 si está presente en el documento o en la pregunta, y 0 si no lo está (Salton y McGill, 1983). 
De esta forma, si en un SID se utilizaran sólo seis únicos términos, $t_{1}, t_{2}, t_{3}$, $t_{4}, t_{5}$ y $t_{6}$, un documento $D_{1}$ que poseyera los términos $t_{1}, t_{3}, t_{4}, t_{5}$, se representaría con el siguiente vector: $(1,0,1,1,1,0)$, mientras que un documento $D_{2}$ que poseyera los términos $t_{2}$ y $_{6}$ se representaría como: $(0,1,0,0,0,1)$. Por su parte, una pregunta $P_{1}$ que se supone indizada con los términos $t_{1}, t_{4}, t_{5}$, se representaría como $(1,0,0,1,1,0)$.

La función de comparación, entonces, es una función que estima, para cada documento de la base, en qué medida se asemeja a la pregunta formulada, calculando, por ejemplo, cuántos términos posee en común con la pregunta y seleccionando aquellos documentos que superan un cierto umbral. Por ejemplo, dada la pregunta $P_{1}$, puede calcularse que el documento $D_{1}$ exhibe un mayor grado de semejanza que $\mathrm{D}_{2}$ (Salton y McGill, 1983; Frakes y Baeza-Yates, 1992).

Como respuesta, el usuario, al examinar los documentos entregados por el sistema, puede juzgar a algunos documentos más relevantes que otros, a partir de lo cual la función de comparación puede lanzar una nueva operación utilizando, en esta ocasión, los atributos de los documentos más relevantes como modelo de búsqueda. El proceso puede iterarse tantas veces como sea necesario, hasta que el usuario estime que ya ha localizado los documentos que satisfacen su necesidad de información.

En este modelo, los términos del documento (y los términos de la pregunta) son palabras del lenguaje natural, que se pueden obtener bien por derivación o bien por asignación. Por derivación significa que los términos se toman del propio documento sin utilizar ninguna fuente externa de conocimiento. Por asignación significa que los términos de indización se asignan mediante la consulta de un lenguaje controlado de indización, como un tesauro.

Las tecnologías que implementan el modelo precedente suelen utilizar únicamente una versión parcial de éste (Codina y Abadal, 1992). Normalmente, suelen utilizar un motor de recuperación de información que trata a los documentos como variables boolenas.

Las variables booleanas sólo pueden ser verdaderas o falsas, con lo cual los sistemas de gestión documental convencionales únicamente pueden crear subconjuntos de documentos relevantes y no relevantes, pero sin establecer grados de relevancia. Sin embargo, en los últimos dos o tres años, han aparecido ya algunos sistemas de gestión documental que incorporan cálculos de relevancia utilizando alguna variación del modelo conceptual vectorial señalado antes (Codina, 1993; Codina, 1994).

\section{Proposiciones sobre los sistemas de información documental}

Proposición general: Los SID son necesarios y son distintos de los sistemas de información convencionales.

Discusión: Los SID son necesarios porque son los únicos sistemas capaces de explotar fondos documentales no triviales.

Los sistemas de información convencionales (no documentales) no son suficientes o no son adecuados para gestionar documentos cognitivos, porque los sistemas convencionales están diseñados para modelar sistemas de actividades, mientras que 
los SID están diseñados para modelar sistemas de conocimiento. La información puede automatizar un SID, pero no puede aportar la lógica del SID, sino que ésta es aportada por la Documentación.

En cambio, los SID se inspiran en los métodos y los procedimientos tecnológicos, científicos y racionales que estudia la disciplina científica conocida con el nombre de Documentación (o Ciencias de la Documentación).

Para gestionar esa clase de fondos documentales, que la humanidad produce sin cesar, la mera intuición no es suficiente. La técnicas documentales no son intuitivas, ni se desprenden directamente de otros conocimientos fundamentales, como las Matemáticas, la Historia o la Física. Por la misma razón, no se derivan tampoco de la Informática o de las Ciencias de la Computación.

Justificación alternativa: Los documentos cognitivos constituyen la memoria social de la humanidad, el registro de todos sus logros científicos y culturales, así como la memoria que registra su pasado y su historia. Un fondo de documentos cognitivos es, de hecho, un depósito de conocimientos que, convenientemente explotado, puede ser puesto a disposición de toda la sociedad.

Un sistema de información debe modelar adecuadamente a su sistema objeto, so pena de ineficiencia. El sistema objeto de un sistema convencional es un sistema de actividades (por ejemplo, las actividades de una empresa); mientras que el sistema objeto de un SID es un depósito de conocimientos.

Proposición particular 1: Los SID son sistemas probabilistas, y no deterministas, y esa circunstancia es una de las que más poderosamente marcan a los SID, en contraste con los sistemas de información convencionales.

Discusión: Los SID son probabilísticos porque dado un documento cognitivo, es imposible saber por cual de sus atributos será necesario recuperarlo. No hay más remedio que representar exhaustivamente tanto sus atributos estructurales (título, autor, lugar de publicación, etc.) como los semánticos (tema, contenido, enfoque, alcance del documento, etc.). Además, no existe ningún algoritmo que determine cuál es la representación más adecuada de un documento, es decir, sobre qué temas trata un documento, cuántos, en qué grado, con qué términos se expresarán mejor los conceptos que trata, etc.

Dada una necesidad de información, es imposible saber, a priori, qué documentos resultarán relevantes, puesto que la relevancia de un documento es una coproducción entre las características del documento y las características subjetivas del usuario. Además, las necesidades de información son inobservables, y no existe ningún algoritmo que determine con precisión absoluta cuál es su representación exacta, con qué conceptos se representa, con qué términos se representan los conceptos, etc.

Proposición particular 2: El objeto de estudio de la Documentación es la representación, el almacenamiento, recuperación y difusión de la información registrada en documentos cognitivos, y su propósito es producir sistemas eficientes de información documental para poner el conocimiento producido por la humanidad a disposición de la humanidad. En consecuencia, la Documentación estudia las características y propiedades de los SID, así como los problemas de análisis, diseño e implantación de los SID.

Discusión: La información es objeto de estudio de muchas disciplinas: la Informática, las Telecomunicaciones, la Lógica, la Semiótica, la Lingüística, etc., pero 
cada una de ellas se atiene a un aspecto o manifestación diferente de la información. A la Documentación le corresponde estudiar el aspecto señalado, que no es objeto de estudio, de facto, de ninguna otra disciplina. Dicho de otra manera: el estudio de la representación, almacenamiento, recuperación y difusión de los conocimientos registrados en documentos requiere la existencia de una disciplina cientifica específica, llámese Documentación o X.

\section{Bibliografia}

BAIGET, T., 1986. Análisis de sistemas de información (apuntes del curso: Organización de los sistemas de Documentación en la Empresa). Barcelona: Institut Català de Tecnologia, 1986.

BENYON, D., 1990. Information and data modelling. Oxford: Blackwell Scientific Publications, 1990.

BERTALANFFY, L. von., 1986. Teoria general de los sistemas. México: Fondo de Cultura Económica, 1986.

BLAIR, D. C., 1990. Language and representation in information retrieval. Amsterdam: Elsevier, 1990.

BUCKLAND, M., 1991. Information and information systems. Westport: Greenwood Press, 1991.

BUNGE, M., 1993. Social systems. En Rodríguez Delgado, R.; Banathy, B. H. International systems science handbook: an introduction to systems science for everybody. Madrid: Systemic Publication, 1993, 211-221.

CANALS, 1., 1994. Los documentos compuestos vivos, base de los futuros sistemas orientados al documento. Information World en Español, 25, junio 1994, 14-16.

CODINA, L., 1993. Nuevos programas de gestión documental para microordenadores: Informe especial. Information World en Español, 18 octubre 1993, 18-19.

CODINA, L., 1984. Bases de datos documentales: estado actual de la tecnología y estrategias de utilización (I). Binary, junio 1994 (en prensa).

CODINA, L., y ABADAL, E., 1992. Gestió documental amb microordinadors: característiques, estructura i tecnologia dels sistemes de gestió documental. Item: Revista de Biblioteconomia i Documentació, 11, juliol-desembre 1992, 72-100.

CURRAS, E., 1988. La información en sus nuevos aspectos. Madrid: Paraninfo, 1988.

CHECKLAND, P. B., 1981. Systems thinking, systems practice. Chichester: Wiley, 1981.

ELLIS, D., 1990. New horizons in information retrieval. London: The Library Association, 1990.

FRAKES, W. B., y BAEZA-YATES, R., 1992. Information retrieval: data structures and algorithms. Englewood Cliffs: Prentice-Hall, 1992.

GUINCHAT, C., y MENOU, M., 1992. Introducción general a las ciencias y técnicas de la información y documentación (segunda edición en francés corregida y aumentada por Blanquet, M. F.; edición en español traducida por el CINDOC). Madrid: CINDOC, UNESCO, 1992.

JACKSON, G. A., 1990. Introducción al diseño de bases de datos relacionales. Madrid: Anaya, 1990.

MACLEOD, I. A., 1991. Text Retrieval and the Relational Model. Journal of the American Society for Information Science (42), 3, 1991, 155-165.

MORON, M. Sistemes experts i Documentació. Item: Revista de Biblioteconomía i Documentació, 2-3, ener-december, 1988, 9-18.

SALTON, G., y MCGILL, M. J., 1983. Introduction to Modern Information Retrieval. Tokyo: McGraw-Hill, 1983. 
SOERGEL, D., 1985. Organizing information: principles of data base and retrieval systems. Orlando: Academic Press, 1985.

VAN GIGCH, J. P., 1987. Teoría general de sistemas. México: Trillas, 1987.

VAN SLYPE, G., 1988. Documentologie. Bruxelles: Presses Universitaires de Bruxelles, 1988.

WALKER, D. W., 1991. Sistemas de información basados en ordenador. Barcelona: Marcombo, 1991.

WILLITTS, J., 1992. Database design and construction: an open learning course for students and information managers. London: Library Association Publishing, 1992.

YOURDON, E., 1993. Análisis estructurado moderno. México: Prentice-Hall Hispanoamericana, 1993. 